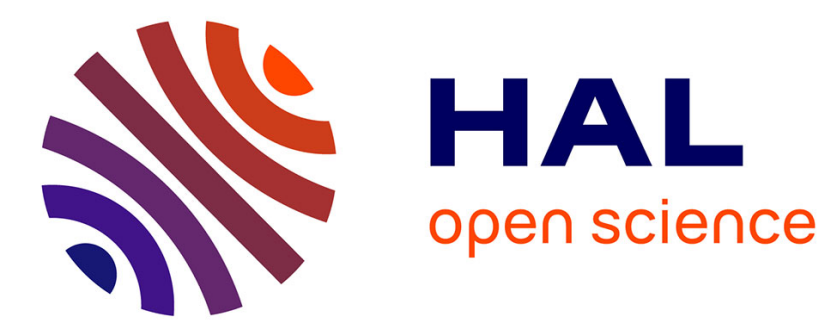

\title{
High gain observer design for some networked control systems
}

Tarek Ahmed-Ali, Françoise Lamnabhi-Lagarrigue

\section{To cite this version:}

Tarek Ahmed-Ali, Françoise Lamnabhi-Lagarrigue. High gain observer design for some networked control systems. IEEE Transactions on Automatic Control, 2012, 57 (4), pp.995-1000. 10.1109/TAC.2011.2168049 . hal-00648730

\section{HAL Id: hal-00648730 \\ https://hal-centralesupelec.archives-ouvertes.fr/hal-00648730}

Submitted on 6 Jul 2021

HAL is a multi-disciplinary open access archive for the deposit and dissemination of scientific research documents, whether they are published or not. The documents may come from teaching and research institutions in France or abroad, or from public or private research centers.
L'archive ouverte pluridisciplinaire $\mathbf{H A L}$, est destinée au dépôt et à la diffusion de documents scientifiques de niveau recherche, publiés ou non, émanant des établissements d'enseignement et de recherche français ou étrangers, des laboratoires publics ou privés. 


\section{High Gain Observer Design for Some Networked Control Systems}

\author{
Tarek Ahmed-Ali and Françoise Lamnabhi-Lagarrigue
}

\begin{abstract}
New results on high gain observer design for networked control systems via an emulation-like approach are presented. By using a general framework and a Lyapunov approach, we derive some explicit conditions on the maximum allowable transmission interval that ensure an exponential convergence of the observation error for a large class of network protocols.
\end{abstract}

Index Terms-High gain observer, networked control systems (NCSs), time-delay systems.

\section{INTRODUCTION}

The control and observation of systems called Networked control systems (NCSs) are currently attracting a lot of attention in the control community. In many applications, the interest for NCSs is motivated by many advantages they offer such as the ease of maintenance and installation, the greater flexibility and the low cost. For these reasons, many industrial control applications use a serial communication channel to connect sensors and controllers. In NCSs, the serial communication channel has many nodes (sensors and actuators) but the signals of these nodes cannot be transmitted at the same time. The rule that selects which node will use the network to transmit its data, is

T. Ahmed-Ali is with GREYC UMR CNRS 6072, Caen Cedex 14050, France (e-mail: tarek.ahmed-ali@greyc.ensicaen.fr).

F. Lamnabhi-Lagarrigue is with CNRS, Laboratoire des Signaux et Systemes (INSIS-INS2I), European Embedded Control Institute (EECI) SUPELEC, Gifsur-Yvette 91192, France (e-mail: lamnabhi@1ss.supelec.fr). called scheduling network protocol. In the present letter, only communication constraints on sensors are considered. It is well known that the stability of these systems is largely determined by the transmission protocol used and by the so-called maximum allowable transfer interval (MATI), i.e., the maximum allowable time between any two successive transmissions in the network. There exist several families of protocols in the literature, but in this study, only Uniformly Globally Exponentially Stable (UGES) protocols are considered. This class includes several protocols, such Round Robin (RR), Try-Once-Discard (TOD) and sampled data systems. For further details on NCSs, protocols and their properties, see the overview papers [1]-[4].

The present note is focused on the implementation of a class of nonlinear observers on NCSs. Although the control of these systems has received considerable attention in the recent years, only few papers which deal with the observer design problem exist in the literature. For example, in deterministic case, the authors in [5] derived some conditions in order to maintain the observability of discrete-time linear systems under network communications. In [6], the authors proposed a method for the mutual design of protocols and observers for linear systems. The considered protocol gives the priority to the node where the error, between the measure and the estimate is the biggest, according to some weighted norms. Sufficient conditions for quadratic stability properties are then given using matrix inequalities. In [7], the authors consider the design of a linear observer for NCSs in the presence of time delay and sampling phenomenons without considering the constraints introduced by the protocols. They give sufficient conditions guaranteing asymptotic stability, by using a Lyapunov-Krasovskii approach. Recently, a small gain approach was used in [8] and [9] for the observers design via an emulation-like approach for some classes of networked control systems. The results contained in [8] and [9] state that if the observer has some robustness properties with respect to measurements errors then, for sufficiently small MATI, the stability in an appropriate sense of the observation error is guaranteed for several classes of network protocols. On the other hand, even without disturbances, the framework developed in [9] does not allow asymptotic stability of the observation error when the network is in zero order hold $(\mathrm{ZOH})$ fashion. In the present study, we focus on a class of nonlinear triangular systems without considering disturbances. More precisely, we provide a framework and use a Lyapunov approach to guarantee an exponential stability of the observation error and improve the bounds of MATI compared to small gain approaches. We specially show that by using the framework presented below and a suitable Lyapunov-Krasovskii functional, we can derive an exponential stability of the observation error when the network is in $\mathrm{ZOH}$ fashion. Note that the results presented in the present letter can be easily extended to several classes of observers such, for example, linear observers and the class of observers described in [10]. The present note is organized as follows:

In Section II, we present the notations which will be used throughout the letter. The framework that we propose is formulated in Section III. In Section IV, we present our results on the emulation of high gain observers on a class of networks.

\section{NOTATIONS AND PRELIMINARIES}

First some mathematical notations are introduced. Let $\mathbf{R}=$ $(-\infty, \infty), \mathbf{R}_{+}=[0, \infty)$ and $\mathbf{N}$ is the set of natural numbers. The notation $\mathbf{N}^{*}$ denotes the set of strictly positive integers. The usual euclidian norm of any vector $v$ will be noted by $|v|$ and the identity matrix of size $p$ is denoted by $I_{p}$. The matrix $0_{p}$ is a square matrix of size $p$ with all values equal to zero. The notations $\lambda_{\min }(S)$ and $\lambda_{\max }(S)$ denote respectively the minimum and maximum eigenvalues of the square matrix $S$. A continuous function $\Gamma: \mathbf{R}_{+} \rightarrow \mathbf{R}_{+}$ 
is said to be of class $\mathcal{K}$, if it is zero at zero, strictly increasing. It is of class $\mathcal{K}_{\infty}$, if it is of class $\mathcal{K}$ and unbounded. A continuous function $\varphi: \mathbf{R}_{+} \times \mathbf{R}_{+} \rightarrow \mathbf{R}_{+}$is said to be of class $-\mathcal{K} \mathcal{L}$, if $\varphi(., t)$ is of class $\mathcal{K}$ for each $t \geq 0$, and $\varphi(s,$.$) is non increasing$ and satisfies $\lim _{t \rightarrow \infty} \varphi(s, t)=0$. The notation $e_{y}\left(a^{+}\right)$represents $\lim _{t \rightarrow a, t>a} e_{y}(t)$ when it exists. The term $W_{\left[t_{0}, t\right)}$ represents ess. $\sup _{s \in\left[t_{0}, t\right)}|W(s)|$. Throughout this letter the vectors $x \in \mathbf{R}^{n}$ and $y \in \mathbf{R}^{p}$ represent respectively the state vector and the outputs of the considered systems.

We consider the following class of nonlinear systems:

$$
\left\{\begin{array}{l}
\dot{x}=A x+f(x) \\
y=C x=x^{1}
\end{array}\right.
$$

where $x=\left(x^{1}, x^{2}, \ldots, x^{q}\right)^{T} \in \mathbf{R}^{n}$ with $x^{k} \in \mathbf{R}^{p}$ and $p . q=n$

$$
A=\left(\begin{array}{ccccc}
0_{p} & I_{p} & 0_{p} & \ldots & 0_{p} \\
0_{p} & 0_{p} & I_{p} & 0_{p} & \vdots \\
\vdots & 0_{p} & \ldots & I_{p} & 0_{p} \\
0_{p} & \vdots & \ldots & 0_{p} & I_{p} \\
0_{p} & \ldots & \ldots & \ldots & 0_{p}
\end{array}\right)
$$

and

$$
C=\left(\begin{array}{llll}
I_{p} & 0_{p} & \ldots & 0_{p}
\end{array}\right)
$$

Throughout this letter, we assume that the following hypotheses are satisfied:

Hypothesis 1: The vector $f(x)$ has the following form:

$$
f(x)=\left(f_{1}\left(x^{1}\right), \ldots, f_{s}\left(x^{1}, \ldots, x^{s}\right), \ldots, f_{q}(x)\right)^{T} .
$$

Hypothesis 2: The functions $f_{s}$ are globally Lipschitz, i.e:

$$
\begin{aligned}
\exists \beta_{0} & >0 \text { such that } \forall\left(x_{1}, x_{2}\right) \in \mathbf{R}^{\mathbf{p} \cdot \mathbf{s}} \times \mathbf{R}^{\mathbf{p . s}} \\
\left|f_{s}\left(x_{1}\right)-f_{s}\left(x_{2}\right)\right| & \leq \beta_{0}\left|x_{1}-x_{2}\right|,(s=1, \ldots, q) .
\end{aligned}
$$

Using these hypotheses, the following observer has been proposed in [11] for the class of systems (1) without considering network:

$$
\dot{\hat{x}}=A \hat{x}+f(\hat{x})-\theta \Delta^{-1} S^{-1} C^{T}(C \hat{x}(t)-y)
$$

where $\theta$ is a positive constant satisfying $\theta \geq 1 . S$ is a symmetric positive definite matrix, solution of the algebraic Lyapunov equation

$$
S A+A^{T} S-C^{T} C=-S
$$

and $\Delta$ is a diagonal matrix which has the following form:

$$
\Delta=\operatorname{Diag}\left(I_{p}, \frac{1}{\theta} I_{p}, \ldots, \frac{1}{\theta^{q-1}} I_{p}\right) .
$$

The exponential convergence of this observer has been derived by using the Lyapunov function $V=\bar{x}^{T} S \bar{x}$, where $\bar{x}=\Delta \tilde{x}$. The authors prove in [11] that its time derivative satisfies

$$
\dot{V} \leq-(\theta-\beta) V-\theta \bar{x}^{T} C^{T} C \bar{x} \quad \text { where } \quad \beta=2 \sqrt{q} \frac{\lambda_{\max }(S)}{\lambda_{\min }(S)} \beta_{0} .
$$

\section{OBSERVERS FOR NETWORKED CONTROL SYSTEMS}

In this section, we present a framework dedicated to implementation of a class of nonlinear observers on a class of networks. Let us consider the nonlinear systems

$$
\left\{\begin{array}{l}
\dot{x}=f_{M}(x) \\
y=h_{M}(x)
\end{array}\right.
$$

and suppose that there exists an observer described by the dynamics

$$
\dot{\hat{x}}=f_{o}\left(\hat{x},\left(h_{M}(\hat{x})-y\right)\right)
$$

observation error $\tilde{x}=\hat{x}-x$ which is represented by the system

$$
\dot{\tilde{x}}=f_{o}\left(\hat{x},\left(h_{M}(\hat{x})-y\right)\right)-f_{M}(x)
$$

is globally asymptotically stable. Now, let us consider the case where all outputs of system (10) are connected to observer (11) via a serial communication channel. We suppose that the signals of these sensors cannot be transmitted to observer (11) at the same time, then this problem can be modeled by the following framework:

$$
\left\{\begin{array}{l}
\dot{x}=f_{M}(x) \quad t \in\left[t_{i-1}, t_{i}\right] \\
x\left(t_{i}^{+}\right)=x\left(t_{i}\right) \\
y=h_{M}(x) \\
\dot{\hat{x}}=f_{o}(\hat{x}, \hat{w}-w) \quad t \in\left[t_{i-1}, t_{i}\right] \\
\hat{x}\left(t_{i}^{+}\right)=\hat{x}\left(t_{i}\right) \\
\dot{w}=g_{w}(w, \hat{w}, \hat{x}) \quad t \in\left[t_{i-1}, t_{i}\right] \\
w\left(t_{i}^{+}\right)=y_{s}\left(t_{i}\right)+h_{y}\left(i, e_{y}\left(t_{i}\right)\right) \\
\dot{\hat{w}}=\hat{g}_{\hat{w}}(\hat{w}, w, \hat{x}) \quad t \in\left[t_{i-1}, t_{i}\right] \\
\hat{w}\left(t_{i}^{+}\right)=h_{M}\left(\hat{x}\left(t_{i}\right)\right) \\
\dot{y}_{s}=g_{y_{s}}\left(y_{s}, x\right) \quad t \in\left[t_{i-1}, t_{i}\right) \\
y_{s}\left(t_{i}\right)=y\left(t_{i}\right) \quad i \in \mathbf{N}^{*} \\
e_{y}=w-y_{s}
\end{array}\right.
$$

The vector $\hat{x}$ is the continuous-time estimate of the system state $x$. The monotonically increasing sequence $t_{j}, j \in \mathbf{N}$ represents the transmission instants. The vectors $w$ and $\hat{x}$ (which are re-initialized at each instant $t_{j}$ ) represent respectively most recently transmitted output values via network and the prediction of the observer output. The vector $y_{s}$ (which is also re-initialized at $t_{j}$ ) allows us the possibility to describe the sampling phenomena. More precisely, $y_{s}$ can be used to represent the output of the zero order hold $(\mathrm{ZOH})$ device. This variable is specially useful when the network operates in $\mathrm{ZOH}$ fashion. In this case we replace conjointly the outputs of the system and the observer by their sampled signals before considering the constraints introduced by the network. As, we will see in the sequel, this framework allows us to derive an asymptotic stability of the observation error when the network operates in $\mathrm{ZOH}$ fashion. The functions $g_{y_{s}}, g_{w}$ and $\hat{g}_{\hat{x}}$ represent prediction functions between two transmission instants. The error induced by the network is represented by the vector $e_{y}$. The protocol $h_{y}$ is the algorithm by which the access to network of each node is determined. At each $t_{j}$, the protocol $h_{y}$ selects which nodes $k \in\{1, \ldots, l\}$ can transmit its data throughout the network. This algorithm is represented by the discrete time system: $e_{y}\left(t_{j}^{+}\right)=h_{y}\left(j, e_{y}\left(t_{j}\right)\right) j \in \mathbf{N}$. Throughout this note, the variable $\tau=\max \left(t_{j+1}-t_{j}\right)$ represents the maximum allowable transfer interval (MATI) and $\tau_{M A T I}$ represents an upper bound of $\tau$. To prevent zeno solution we suppose that the MATI satisfies $\tau>t_{j+1}-t_{j}>\mu$, where $\mu$ is an arbitrary positive constant. Note that the sampled-data systems are a special case of NCSs since all sensor and control signals are transmitted at each transmission instant. In this case, the protocol $h_{y}$ is equal to zero, and the maximum allowable time between any two successive transmissions is called maximum allowable sampling period MASP. Compared to [12] where a framework based on discrete-time approximate models is developed for the observer design for sampled-data systems, we can say that the framework (13) is an alternative approach to [12].

Hypothesis 3: We suppose that the protocol $h_{y}$ is $U G E S$. This means that there exists a positive function, $W: \mathbf{N} \times \mathbf{R}^{n_{e y}} \rightarrow \mathbf{R}_{+}$and some 
positive constants $\lambda \in[0,1), a_{1}, a_{2}$ such that, for all $j \in \mathbf{N}$ and for all $e_{y} \in \mathbf{R}^{n_{e y}}$

$$
\begin{aligned}
a_{1}\left|e_{y}\right| & \leq W\left(j, e_{y}\right) \leq a_{2}\left|e_{y}\right| \\
W\left(j+1, h_{y}\left(j, e_{y}\right)\right) & \leq \lambda W\left(j, e_{y}\right) .
\end{aligned}
$$

Hypothesis 4: We also assume that there exists a positive constant $M_{0}$ so that for all $j \in \mathbf{N}$ and for all $e_{y} \in \mathbf{R}^{n_{e y}}$

$$
\left|\frac{\partial W\left(j, e_{y}\right)}{\partial e_{y}}\right| \leq M_{0}
$$

This condition is satisfied by many protocols such Round Robin (RR) and Try-Once-Discard (TOD).

\section{HIGH GAIN OBSERVER FOR NCSS}

In this section we focus on the implementation of high gain observer (6) over a network with UGES protocols. We present two examples: The first one is an implementation in $\mathrm{ZOH}$ fashion, whereas the second one, is based on the introduction of an output predictor between two transmission instants. In both cases we prove exponential convergence of the high gain observer for sufficiently small MATI.

\section{A. High Gain Observer in ZOH Fashion}

When the network operates in $\mathrm{ZOH}$ fashion, we will consider that all prediction functions in (13) are equal to zero. This means that the corrector term $\hat{w}-w$ is held constant between two transmission instants. Following framework (13), we propose this observer:

$$
\left\{\begin{array}{l}
\dot{\hat{x}}=A \hat{x}+f(\hat{x})-\theta \Delta^{-1} S^{-1} C^{T}(\hat{w}-w) \\
\dot{w}=0 \quad t \in\left[t_{i-1}, t_{i}\right] \\
w\left(t_{i}^{+}\right)=y_{s}\left(t_{i}\right)+h_{y}\left(i, e_{y}\left(t_{i}\right)\right) \\
\dot{\hat{w}}=0 \quad t \in\left[t_{i-1}, t_{i}\right] \\
\hat{w}\left(t_{i}^{+}\right)=C \hat{x}\left(t_{i}\right) \\
\dot{y}_{s}=0 \quad t \in\left[t_{i-1}, t_{i}\right) \\
y_{s}\left(t_{i}\right)=y\left(t_{i}\right) \\
e_{y}=w-y_{s}
\end{array}\right.
$$

if we consider the observation error $\tilde{x}=\hat{x}-x$, then we have

$$
\left\{\begin{array}{l}
\dot{\tilde{x}}=\left(A-\theta \Delta^{-1} S^{-1} C^{T} C\right) \tilde{x}+f(\tilde{x}+x)-f(x) \\
-\theta \Delta^{-1} S^{-1} C^{T}\left(C \tilde{x}\left(t_{i-1}^{+}\right)-C \tilde{x}(t)-e_{y}\right) \quad t \in\left[t_{i-1}, t_{i}\right] \\
\dot{e_{y}}=0 \quad t \in\left[t_{i-1}, t_{i}\right] \\
\tilde{x}\left(t_{i}^{+}\right)=\tilde{x}\left(t_{i}\right) \\
e_{y}\left(t_{i}^{+}\right)=h_{y}\left(i, e_{y}\left(t_{i}\right)\right)
\end{array}\right.
$$

As we can see, system (18) is disturbed by the term $C \tilde{x}\left(t_{i-1}^{+}\right)-C \tilde{x}(t)$. The Leibniz-Newton formula Provides

$$
C \tilde{x}\left(t_{i-1}^{+}\right)-C \tilde{x}(t)=-C \int_{t-\tau_{1}(t)}^{t} \dot{\tilde{x}}\left(s_{1}\right) d s_{1}
$$

with $\tau_{1}(t)=t-t_{i-1}^{+}$for all $t \in\left(t_{i-1}, t_{i}\right]$. Note that we have $\tau_{1}(t) \in$ $[0, \tau]$ and $\dot{\tau}_{1}=1$. This means that system (18) can be viewed as a time delay system with bounded time varying delay. This allows us to extend the approach developed in [4], [13] and [14] to the problem considered in the present note.

Theorem 1: Let us consider system (1) and suppose that hypotheses $1,2,3,4$ hold. Then, there exists a positive constant $\theta_{0}$, such that for all $\theta>\theta_{0}$ system (17) is a global exponential observer for system
(1) if $\tau \in\left[\mu, \tau_{M A T I}\right)$ where $\tau_{M A T I}=\min \left(\lambda_{\min }(S) / 6 \sup \{[\mid A-\right.$ $\left.\left.\left.S^{-1} C^{T} C \mid+\sqrt{q} \beta_{0}\right], \lambda_{\min }(S) \lambda_{\max }\left(S^{-1}\right)\right\}^{2} \theta, 1-\lambda\right)$.

Proof: Consider the change of coordinates $\bar{x}=\Delta \tilde{x}$, and from the fact that $\Delta A \Delta^{-1}=\theta A$ and $C^{T} C \Delta^{-1}=C^{T} C$, then (18) will be

$$
\left\{\begin{array}{l}
\dot{\bar{x}}=\theta\left(A-S^{-1} C^{T} C\right) \bar{x}+\Delta(f(\tilde{x}+x)-f(x)) \\
+\theta S^{-1} C^{T}\left(e_{y}+C \int_{t-\tau_{1}(t)}^{t} \dot{\bar{x}}\left(s_{1}\right) d s_{1}\right) t \in\left[t_{i-1}, t_{i}\right] \\
\dot{e_{y}}=0 \quad t \in\left[t_{i-1}, t_{i}\right] \\
e_{y}\left(t_{i}^{+}\right)=h_{y}\left(i, e_{y}\left(t_{i}\right)\right)
\end{array}\right.
$$

In order to prove this theorem, let us consider the following candidate Lyapunov-Krasovskii functional:

$$
U_{1}=\bar{x}^{T} S \bar{x}+\int_{t-\tau_{M A T I}}^{t} \int_{s}^{t}\left|\dot{\bar{x}}\left(s_{1}\right)\right|^{2} d s_{1} d s+\gamma_{1} \phi_{1}(t) W^{2}\left(j, e_{y}\right)
$$

where $\gamma_{1}>0, j \in \mathbf{N}$ and $\phi_{1}(t)$ is a bounded, positive function and decreasing between $\left(t_{i-1}^{+}, t_{i}\right]$, with appropriate fixed values at instants $t_{i-1}^{+}$and $t_{i}$. The functional $U_{1}$ has been used in [4], [13] and [14] for observer design with sampled and delayed measurements without network constraints communications by considering $W^{2}\left(j, e_{y}\right)=0$. We will show below that this approach can be used here. More precisely, to prove exponential stability, it is sufficient to find $\tau_{M A T I}$ and $\theta_{0}$ so that for $\theta>\theta_{0}$ the following inequalities hold:

$$
\left\{\begin{array}{l}
\dot{U}_{1}+\varepsilon_{1} U_{1} \leq 0 \quad t \in\left(t_{i-1}, t_{i}\right] \\
U_{1}\left(t_{i}^{+}\right)<U_{1}\left(t_{i}\right) \quad \forall i \in \mathbf{N}^{*}
\end{array}\right.
$$

where $\varepsilon_{1}>0$. We can easily see that the second inequality of (22) can be satisfied by choosing $\phi_{1}\left(t_{i-1}^{+}\right)=1$ and $\phi_{1}\left(t_{i-1}^{+}+\tau_{M A T I}\right)=$ $\lambda \forall i \in \mathbf{N}^{*}$ where $\lambda \in(0,1)$.

Let us compute the derivative of $U_{1}$ for $t \in\left(t_{i-1}, t_{i}\right]$, then we have

$$
\begin{aligned}
\dot{U}_{1}=2 \bar{x}^{T} S \dot{\bar{x}}+\tau_{M A T I}|\dot{\bar{x}}|^{2}-\int_{t-\tau_{M A T I}}^{t}|\dot{\bar{x}}(s)|^{2} d s & \dot{\gamma}_{1}(t) W^{2}\left(i-1, e_{y}\right) .
\end{aligned}
$$

Using (20), and Hölder inequality we can deduce that

$$
|\dot{\bar{x}}|^{2} \leq 2 k_{2} \theta^{2}\left[V+|I|^{2}+\frac{W^{2}\left(i-1, e_{y}\right)}{a_{1}^{2}}\right]
$$

where

$$
k_{2}=\frac{3 \sup \left\{\left[\left|A-S^{-1} C^{T} C\right|+\sqrt{q} \beta_{0}\right], \lambda_{\min }(S) \lambda_{\max }\left(S^{-1}\right)\right\}^{2}}{2 \lambda_{\min }(S)} .
$$

Now, let us set $V=\bar{x}^{T} S \bar{x}$, after some computations we deduce that for $t \in\left(t_{i-1}, t_{i}\right]$, we have

$$
\begin{aligned}
\dot{U}_{1}+\varepsilon_{1} U_{1} \leq & -\theta V+\beta V+\varepsilon_{1} V+2 \theta\left(|I|^{2}+\frac{W^{2}\left(i-1, e_{y}\right)}{a_{1}^{2}}\right) \\
& +2 \tau_{M A T I} \theta^{2} k_{2}\left[V+|I|^{2}+\frac{W^{2}\left(i-1, e_{y}\right)}{a_{1}^{2}}\right] \\
& +\varepsilon_{1} \tau_{M A T I} \int_{t-\tau_{M A T I}}^{t}|\dot{\bar{x}}(s)|^{2} d s \\
& -\int_{t-\tau_{M A T I}}^{t}|\dot{\bar{x}}(s)|^{2} d s \\
& +\left[\gamma_{1} \dot{\phi}_{1}(t)+\varepsilon_{1} \gamma_{1} \phi_{1}(t)\right] W^{2}\left(i-1, e_{y}\right)
\end{aligned}
$$


where $I=\int_{t-\tau_{1}(t)}^{t} \dot{\bar{x}}(s) d s$. From Jensen's inequality, we can derive

$$
|I|^{2} \leq \tau_{M A T I} \int_{t-\tau_{M A T I}}^{t}|\dot{\bar{x}}(s)|^{2} d s .
$$

By choosing

$$
\dot{\phi}_{1}=-\varepsilon_{1} \phi_{1}-1 \forall t \in\left(t_{i-1}, t_{i}\right] \text { and } \gamma_{1}=\frac{a_{1}^{2}}{2 \theta+2 \tau_{M A T I} \theta^{2} k_{2}}
$$

we can say that the inequality $\dot{U}_{1}+\varepsilon_{1} U_{1} \leq 0$ is ensured, if $\tau_{M A T I}$ satisfies both (27) and the following inequalities:

$$
\left\{\begin{array}{l}
\theta-\beta-\epsilon_{1}-2 \tau_{M A T I} \theta^{2} k_{2}>0 \\
1-2 \theta^{2} k_{2} \tau_{M A T I}^{2}-\epsilon_{1} \tau_{M A T I}-2 \tau_{M A T I} \theta>0
\end{array} .\right.
$$

From this, and by integrating (27) on $\left(t_{i-1}, t_{i-1}+\tau_{M A T I}\right]$ and setting $\epsilon_{1} \rightarrow 0$, we derive the following conditions:

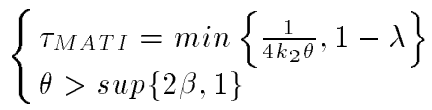

using the fact that $|\bar{x}| \leq\left(1 / \sqrt{\lambda_{\min }(S)}\right) \sqrt{U_{1}(t)}$ and from (22) then we deduce that under conditions (29) there exist $c_{1}>0$ and $c_{2}>0$ such that $|\bar{x}| \leq c_{1} \exp ^{-c_{2}\left(t-t_{0}\right)} \forall t \geq t_{0}$. From this we can say that $|\bar{x}|$ converges exponentially towards zero.

\section{B. High Gain Observer With an Output Predictor}

The idea of using a predictor of the output was introduced in [15] for the design of sampled-data observers. More precisely, this predictor is used by the observer between two transmission instants and its model is a copy of the output system model. This gives this differential equation $\dot{w}=\left(\partial h_{M}(\hat{x}) / \partial \hat{x}\right) f_{M}(\hat{x})$ where the initial condition $w\left(t_{0}\right)$ is arbitrary and at each transmission instant $t_{i}$ the value of $w$ is reseated like this: $w\left(t_{i}^{+}\right)=y\left(t_{i}\right)$. This idea has been extended to networked systems in [8] and [9] and some bounds of MATI have been derived using small gain approach. The aim of this section is to improve the bound of MATI for high gain observer when an output predictor is used. Following the framework (13), we can write the following observer:

$$
\left\{\begin{array}{l}
\dot{\hat{x}}=A \hat{x}+f(\hat{x})-\theta \Delta^{-1} S^{-1} C^{T}(\hat{w}-w) \\
\dot{w}=\hat{x}^{2}+f_{1}\left(\hat{x}^{1}\right) \quad t \in\left[t_{i-1}, t_{i}\right] \\
w\left(t_{i}^{+}\right)=y_{s}\left(t_{i}\right)+h_{y}\left(i, e_{y}\left(t_{i}\right)\right) \\
y_{s}=y \\
\hat{w}=C \hat{x}(t)
\end{array}\right.
$$

Let us consider the observation error $\tilde{x}=\hat{x}-x$, then we have

$$
\left\{\begin{array}{l}
\dot{\tilde{x}}=\left(A-\theta \Delta^{-1} S^{-1} C^{T} C\right) \tilde{x}+f(\tilde{x}+x)-f(x) \\
+\theta \Delta^{-1} S^{-1} C^{T} e_{y} \quad t \in\left[t_{i-1}, t_{i}\right] \\
\dot{e_{y}}=\tilde{x}^{2}+f_{1}\left(\tilde{x}^{1}+x^{1}\right)-f_{1}\left(x^{1}\right) \quad t \in\left[t_{i-1}, t_{i}\right] \\
\tilde{x}\left(t_{i}^{+}\right)=\tilde{x}\left(t_{i}\right) \\
e_{y}\left(t_{i}^{+}\right)=h_{y}\left(i, e_{y}\left(t_{i}\right)\right)
\end{array}\right.
$$

Theorem 2: Let us consider system (1) and suppose that hypotheses $1,2,3,4$ hold. Then for all $\theta>\max \{\beta, 1\}$, system (30) is a global exponential observer for system (1) if $\tau \in\left[\mu, \tau_{M A T I}\right)$ where $\tau_{M A T I}=a_{1}((\pi / 2)-2 \operatorname{atan}(\lambda)) \sqrt{\lambda_{\min }(S)(\theta-\beta)} / M_{0}\left(\theta+\beta_{0}\right) \sqrt{\theta}$.
Proof: As in the above section, using the same change of coordinates then (31) will be

$$
\left\{\begin{array}{l}
\dot{\bar{x}}=\theta\left(A-S^{-1} C^{T} C\right) \bar{x}+\Delta(f(\tilde{x}+x)-f(x)) \\
+\theta S^{-1} C^{T} e_{y} \quad t \in\left[t_{i-1}, t_{i}\right] \\
\dot{e_{y}}=\theta \bar{x}^{2}+f_{1}\left(\tilde{x}^{1}+x^{1}\right)-f_{1}\left(x^{1}\right) \quad t \in\left[t_{i-1}, t_{i}\right] \\
e_{y}\left(t_{i}^{+}\right)=h_{y}\left(i, e_{y}\left(t_{i}\right)\right)
\end{array}\right.
$$

Let us consider the following candidate Lyapunov function as in [16]:

$$
U(t)=\sigma \bar{x}^{T}(t) S \bar{x}(t)+\gamma \phi(t) W^{2}\left(j, e_{y}(t)\right)
$$

where $j \in \mathbf{N}, \sigma$ and $\gamma$ are two positive constants which will be computed below. The positive function $\phi(t)$ is bounded and decreasing on the transmission interval $\left(t_{i-1}, t_{i}\right] i \in \mathbf{N}^{*}$. This function satisfies the following conditions:

$$
\phi\left(t_{i-1}^{+}\right)=\lambda^{-1} \quad \text { and } \quad \phi\left(t_{i-1}^{+}+\tau_{M A T I}\right)=\lambda \quad \forall i \in \mathrm{N}^{*}
$$

where $\lambda \in(0,1)$, to guarantee that $U\left(t_{i}^{+}\right) \leq U\left(t_{i}\right)$. Now, let us compute the time derivative of $U$ between $\left(t_{i-1}, t_{i}\right]$, then we have

$\dot{U}=2 \sigma \bar{x}^{T} S \dot{\bar{x}}+\gamma \dot{\phi} W^{2}\left(i-1, e_{y}\right)+2 \gamma \phi W\left(i-1, e_{y}\right) \dot{W}\left(i-1, e_{y}\right)$

after some computations, we deduce that

$$
\begin{aligned}
\dot{U} \leq & -\sigma(\theta-\beta) \lambda_{\min }(S)|\bar{x}|^{2}+\sigma \theta\left|e_{y}\right|^{2} \\
& +\gamma \dot{\phi} W^{2}\left(i-1, e_{y}\right)+2 \gamma \phi W\left(i-1, e_{y}\right)\left|\frac{\partial W\left(i-1, e_{y}\right)}{\partial e_{y}}\right|\left|\dot{e}_{y}\right| .
\end{aligned}
$$

Let us remark that

$$
\left|\dot{e}_{y}\right| \leq \theta\left|\bar{x}^{2}\right|+\left|f_{1}\left(\hat{x}^{1}\right)-f_{1}\left(x^{1}\right)\right| \leq\left(\theta+\beta_{0}\right)|\bar{x}|
$$

using the property (16), we derive the following inequalities $\mid \partial W(i-$ $\left.1, e_{y}\right) / \partial e_{y} \| \dot{e}_{y}\left|\leq M_{0}\left(\theta+\beta_{0}\right)\right| \bar{x} \mid$ and

$$
\begin{aligned}
\dot{U} \leq-\sigma(\theta-\beta) \lambda_{\min }(S)|\bar{x}|^{2}+\sigma \theta\left|e_{y}\right|^{2} & \\
& +\gamma \dot{\phi} W^{2}\left(i-1, e_{y}\right)+2 \gamma \phi W\left(i-1, e_{y}\right) M_{0}\left(\theta+\beta_{0}\right)|\bar{x}| .
\end{aligned}
$$

Using the fact that the protocol $h_{y}$ is UGES, and by adding and subtracting the term $\epsilon_{0} W^{2}\left(i-1, e_{y}\right)$, where $\epsilon_{0}>0$, thus we have

$$
\begin{aligned}
\dot{U} \leq & -\sigma(\theta-\beta) \lambda_{\min }(S)|\bar{x}|^{2}-\epsilon_{0} W^{2}\left(i-1, e_{y}\right) \\
& +\frac{\sigma \theta}{a_{1}^{2}} W^{2}\left(i-1, e_{y}\right)+\gamma \dot{\phi} W^{2}\left(i-1, e_{y}\right) \\
& +2 \gamma \phi W\left(i-1, e_{y}\right) M_{0}\left(\theta+\beta_{0}\right)|\bar{x}|+\epsilon_{0} W^{2}\left(i-1, e_{y}\right)
\end{aligned}
$$

If we choose

$$
\dot{\phi}=-\gamma\left(\phi^{2}+1\right) \quad \forall t \in\left(t_{i-1}, t_{i}\right]
$$

thus, we will have

$$
\begin{aligned}
\dot{U} \leq-\sigma(\theta-\beta) & \lambda_{\min }(S)|\bar{x}|^{2}+M_{0}^{2}\left(\theta+\beta_{0}\right)^{2}|\bar{x}|^{2} \\
& -\epsilon_{0} W^{2}\left(i-1, e_{y}\right)-\left[\gamma^{2}-\epsilon_{0}-\frac{\sigma \theta}{a_{1}^{2}}\right] W^{2}\left(i-1, e_{y}\right)
\end{aligned}
$$


where $\epsilon_{0}>0$. Note that the term $\epsilon_{0} W^{2}\left(i-1, e_{y}\right)$ is introduced to guarantee the exponential convergence. Choosing

$$
\left\{\begin{array}{l}
\sigma=\frac{M_{0}^{2}\left(\theta+\beta_{0}\right)^{2}}{(\theta-\beta) \lambda_{\min }(S)}+\frac{\epsilon_{0}}{(\theta-\beta) \lambda_{\min }(S)} \\
\gamma=\sqrt{\frac{\sigma \theta}{a_{1}^{2}}+\epsilon_{0}}
\end{array}\right.
$$

thus we can say that there exists a positive constant $\xi$, so that $\dot{U}(t) \leq$ $-\xi U(t) t \in\left(t_{i-1}, t_{i}\right]$ this means that for all $t \in\left(t_{i-1}, t_{i}\right]$

$$
U(t) \leq U\left(t_{i-1}^{+}\right) \exp ^{-\xi\left(t-t_{i-1}^{+}\right)}
$$

and

$$
U\left(t_{i}\right) \leq U\left(t_{i-1}^{+}\right) \exp ^{-\xi\left(t_{i}-t_{i-1}^{+}\right)}
$$

on the other hand, we have

$$
U\left(t_{i}^{+}\right)=V\left(\bar{x}\left(t_{i}^{+}\right)\right)+\gamma \lambda^{-1} W^{2}\left(e_{y}\left(t_{i}^{+}\right)\right)
$$

using the fact that the protocol is UGES, and $\bar{x}\left(t_{i}^{+}\right)=\bar{x}\left(t_{i}\right)$, then we can write

$$
U\left(t_{i}^{+}\right) \leq V\left(\bar{x}\left(t_{i}\right)\right)+\gamma \lambda W^{2}\left(e_{y}\left(t_{i}\right)\right) \leq U\left(t_{i}\right) \quad i \in \mathbf{N}^{*}
$$

thus, we derive

$$
\begin{aligned}
U(t) \leq U\left(t_{i-1}^{+}\right) \exp ^{-\xi\left(t-t_{i-1}^{+}\right)} & \forall t \in\left(t_{i-1}, t_{i}\right] \\
U\left(t_{i}^{+}\right) \leq U\left(t_{i-1}^{+}\right) \exp ^{-\xi\left(t_{i-t_{i-1}}^{+}\right)} & i \in \mathbf{N}^{*} .
\end{aligned}
$$

Using the fact that $|\bar{x}| \leq\left(1 / \sqrt{\sigma \lambda_{\min }(S)}\right) \sqrt{U(t)}$ and from (41), then we deduce that there exist $\mu_{1}>0$ and $\mu_{2}>0$ such that $|\bar{x}| \leq$ $\mu_{1} \exp ^{-\mu_{2}\left(t-t_{0}\right)} \forall t \geq t_{0}$. This means that $\bar{x}$ converges exponentially towards zero, and the value of $\tau_{M A T I}$ is then derived by integrating (36) on $\left(t_{i-1}, t_{i-1}+\tau_{M A T I}\right]$, with $\epsilon_{0} \rightarrow 0$. This leads to

$$
\begin{aligned}
\tau_{M A T I} & =\lim _{\epsilon_{0} \rightarrow 0}\left(\frac{1}{\gamma}\left[\operatorname{atan}\left(\lambda^{-1}\right)-\operatorname{atan}(\lambda)\right]\right. \\
& =\frac{a_{1}\left(\frac{\pi}{2}-2 \operatorname{atan}(\lambda)\right) \sqrt{\lambda_{\min }(S)(\theta-\beta)}}{M_{0}\left(\theta+\beta_{0}\right) \sqrt{\theta}} .
\end{aligned}
$$

1) Comparison With Small Gain Approach: Let us consider the function $V=\bar{x}^{T} S \bar{x}$, then after simple computations, we can easily derive the following inequality:

$$
\dot{V} \leq-(\theta-\beta) V+\theta \frac{W^{2}}{a_{1}^{2}}
$$

using the comparison lemma, then we will have

$$
|\bar{x}| \leq \sqrt{\frac{V\left(t_{0}\right)}{\lambda_{\min }(S)}} \exp \frac{-(\theta-\beta)}{2}\left(t-t_{0}\right)+\sqrt{\frac{\theta}{\lambda_{\min }(S)(\theta-\beta)}} \frac{W_{\left[t_{0}, t\right)}}{a_{1}}
$$

where $\sqrt{V\left(t_{0}\right) / \lambda_{\min }(S)} \exp ^{((-(\theta-\beta)) / 2)}\left(t-t_{0}\right)$ is a function of class $\mathcal{K} \mathcal{L}$.

Now, let us define, $\tilde{y}=M_{0}\left(\theta+\beta_{0}\right) \bar{x}$, then we derive the gain $\gamma_{0}$ from $W$ to $\tilde{y}$

$$
\gamma_{0}=\frac{M_{0}\left(\theta+\beta_{0}\right)}{a_{1}} \sqrt{\frac{\theta}{\lambda_{\min }(S)(\theta-\beta)}} .
$$

Using (44) and the fact that

$$
\left|\frac{\partial W\left(i-1, e_{y}\right)}{\partial e_{y}}\right|\left|\dot{e}_{y}\right| \leq M_{0}\left(\theta+\beta_{0}\right)|\bar{x}|
$$

then from Theorem 4 in [2] we can say that $\tau_{M A T I}$ is given by

$$
\tau_{M A T I}=\frac{1-\lambda}{\gamma_{0}}=\frac{a_{1}(1-\lambda) \sqrt{\lambda_{\min }(S)(\theta-\beta)}}{M_{0}\left(\theta+\beta_{0}\right) \sqrt{\theta}} .
$$
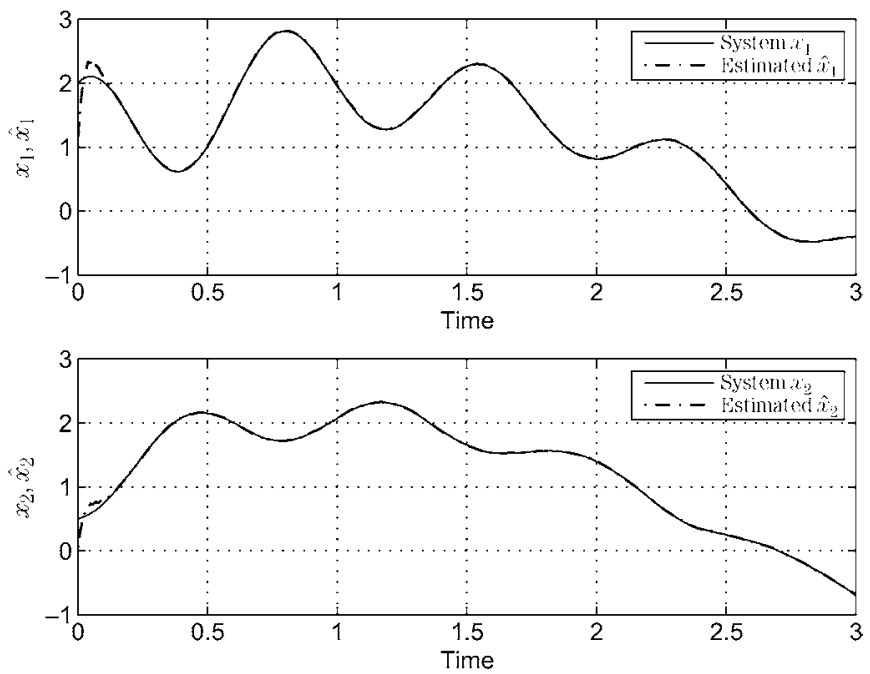

Fig. 1. Evolution of the first and second states for $\tau_{M A T I}=0.01$.

Now let us compute the ration $R$ between (42) and (47), then we have $R=((\pi / 2)-2 \operatorname{atan}(\lambda)) /(1-\lambda)$. Since $1-\lambda \leq(\pi / 2)-2 \operatorname{atan}(\lambda)$ for all $\lambda \in[0,1)$, then we deduce that $R \geq 1$. This means that the bound derived from Lyapunov approach is larger than the one derived from small gain approach. This result means that we have also enlarged the interval of admissible values of the gain $\theta$ for any fixed $\tau_{M A T I}$ and therefore we have also improved the speediness of convergence. Note that in all formulas of $\tau_{M A T I}$, it is clear that $\tau_{M A T I}$ depends inversely on $\theta$. This obviously leads to small values of $\tau_{M A T I}$ for high values of the gain $\theta$.

\section{Simulations}

In this part, we present some simulations on the flexible joint robotic arm used in [13]

$$
\left\{\begin{array}{l}
\dot{x}_{1}=x_{3}+\delta(t) \\
\dot{x}_{2}=x_{4}+\delta(t) \\
\dot{x}_{3}=-48.6 x_{1}+48.6 x_{2}-1.25 x_{3}+21.6 u+\delta(t) \\
\dot{x}_{4}=19.5 x_{1}-19.5 x_{2}-3.3 \sin \left(x_{3}\right)+\delta(t) \\
y_{1}=x_{1} \\
y_{2}=x_{2}
\end{array}\right.
$$

Without unknown uncertainties $\delta(t)$, system (48) satisfies hypotheses $1,2,3,4$ with $p=q=2$ and $x=\left(x^{1}, x^{2}\right)$ where $x^{1}=\left(x_{1}, x_{2}\right)$ and $x^{2}=\left(x_{3}, x_{4}\right)$.

Constrained by the size of the present note, we focused on the simulations of observer (30) with Round-Robin (RR) protocols, that consists in granting access to each compenent of the vector ouputs after $p$ transmission instants where $p$ is the number of ouputs [2]. In the simulations presented below, we also suppose that unknown uncertainties $\delta(t)$ are represented by random function of Matlab with variance $=0.8$. The following simulations are performed with $u=0.1 \sin (t), \theta=40$ and $\tau_{M A T I}=0.01$ (see Figs. 1 and 2).

As we can see, even with some uncertainties, the results remain good with $\theta=40$ and $\tau_{M A T I}=0.01$. This is not a general conclusion. Indeed the uncertainties can cause robustness problems especially for high values of $\theta$.

\section{CONCLUSION}

In this letter, some results on the design of high gain observer for networked systems with UGES protocols were presented. These results can be easily extended to other observers. In future work, we will present some results concerning other classes of protocols. 

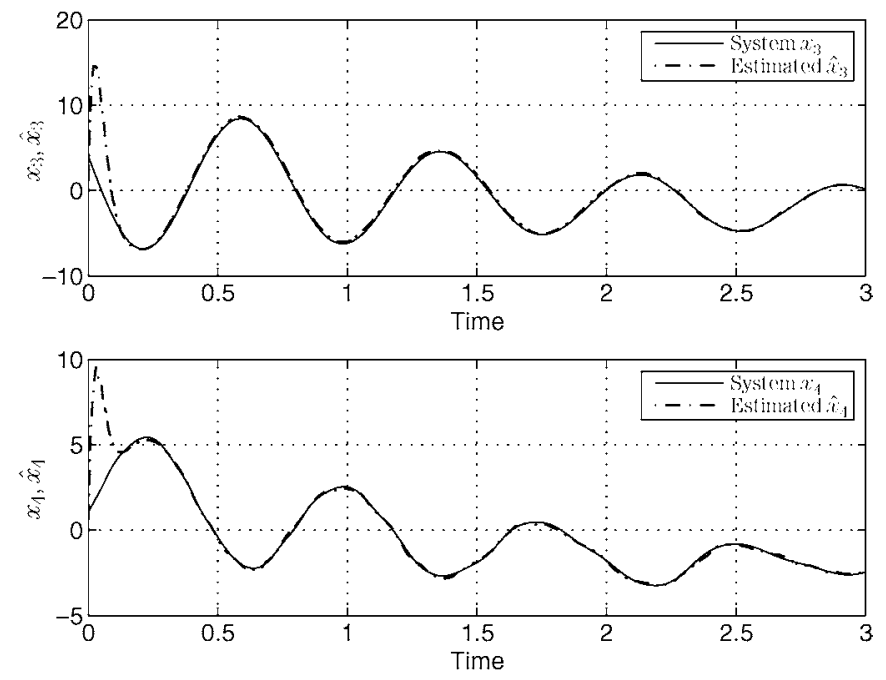

Fig. 2. Evolution of the third and fourth states for $\tau_{M A T I}=0.01$.

\section{REFERENCES}

[1] G. C. Walsh, H. Ye, and G. Bushnell, "Stability analysis of networked control systems," IEEE Trans. Control Sys. Technol, vol. 10, no. 3, pp. 438-446, May 2002.

[2] D. Nešić and A. Teel, "Input-output stability properties of networked control systems," IEEE Trans. Autom. Control, vol. 49, no. 10, pp. 1650-1667, Oct. 2004.

[3] M. Yu, L. Wang, and T. Chu, "Sampled-data stabilization of networked control systems with nonlinearity," Proc. Inst. Elect. Eng., vol. 156, no. 6, pp. 609-614, Nov. 2005.

[4] J. Hespanha, P. Naghshtabrizi, and Y. Xu, "A survey of recent results in networked control systems," IEEE Special Issue Technol. Netw. Control Syst., vol. 95, no. 1, pp. 138-162, 2007.

[5] B. Sinopoli, L. Schenato, M. Franschetti, K. Poolla, M. Jordan, and S. Sastry, "Kalman filtering with intermittent observations," IEEE Trans. Autom. Control, vol. 49, no. 9, pp. 1453-1464, Sep. 2004.

[6] D. Dačić and D. Nešić, "Observer design for wired linear networked control systems using matrix inequalities," Automatica, vol. 44, pp. 2840-2848, 2008.

[7] P. Naghshtabrizi and J. P. Hespanha, "Designing an observer-based controller for a network control system," in Proc. 44th Conf. Decision Control, 2005, [CD ROM].

[8] R. Postoyan, T. Ahmed-Ali, and F. Lamnabhi-Lagarrigue, "Observers for classes of nonlinear networked control systems," in Proc. Conf. Signal Syst. Devices (SSD'09) Djerba-Tunisie, 2009, [CD ROM].

[9] R. Postoyan and D. Nešić, "A framework for the observer design for networked control," in Proc. Amer. Control Conf. (ACC'2010), Baltimore, MD, pp. 3678-3683.

[10] A. Zemouche, M. Boutayeb, and G. I. Bara, "Observers for a class of Lipschitz systems with extension to $\mathcal{H}_{\infty}$ performance analysis," Syst. Control Lett., vol. 57, no. 1, pp. 18-27, 2008.

[11] M. Farza, M. M'Saad, and L. Rossignol, "Observer design for a class of mimo nonlinear systems," Automatica, vol. 40, pp. 135-143, 2004.

[12] M. Arcak and D. Nešić, "A framework for nonlinear sampled-data observer design via approximate discrete-time models and emulation," Automatica, vol. 40, pp. 1931-1938, 2004.

[13] T. Raff, M. Kogel, and F. Allgower, "Observer with sample-and-hold updating for lipschitz nonlinear systems with nonuniformly sampled measurements," in Proc. 48th IEEE-ACC, Shanghai, China, 2008, [CD ROM].

[14] T. Ahmed-Ali, E. Cherrier, and M. MSaad, "Cascade high gain observers for nonlinear systems with delayed output," in Proc. 48th IEEECDC, Shanghai, China, 2009, [CD ROM].

[15] I. Karafyllis and C. Kravaris, "From continuous-time design to sampled-data design of nonlinear observers," IEEE Trans. Autom. Control, vol. 54, no. 9, pp. 2169-2174, Sep. 2009.

[16] D. Carnevale, A. Teel, and D. Nešić, "A Lyapunov proof of an improved maximum allowable transfer interval for networked control systems," IEEE Trans. Autom. Control, vol. 52, no. 5, pp. 892-897, May 2007. 